\title{
Effect of Increasing Running Cadence on Peak Impact Force in an Outdoor Environment
}

\author{
Taylor Musgjerd, DPT ${ }^{1}$, Jacob Anason, DPT ${ }^{1}$, Drew Rutherford, MS ${ }^{1}$, Thomas W Kernozek, PhD, FACSM ${ }^{1}$ (D) \\ 1 Department of Health Professions, University of Wisconsin-La Crosse \\ Keywords: auditory cueing, kinetics, injury, feedback, step rate \\ https://doi.org/10.26603/001c.25166
}

\section{International Journal of Sports Physical Therapy}

Vol. 16, Issue 4, 2021

\section{Background}

An estimated $56 \%$ of recreational runners sustain a running-related injury related to the high impact forces in running. Increasing step frequency (cadence) while maintaining a consistent speed has been shown to be an effective way to lower impact forces which may reduce injury risk.

\section{Purpose}

To examine effects of increased cadence on peak impact force during running in an outdoor setting. It was hypothesized that as cadence increases, peak force would decrease.

\section{Study Design}

Repeated measures, quasi-experimental

\section{Methods}

Peak force and cadence measurements were collected from 15 recreational runners ( 8 females, 7 males) during two 2.4-mile outdoor runs. Peak force was measured using an insole-based load measuring device. Baseline session run was completed at participant's naturally preferred cadence and cadence session run was completed at a cadence targeted to be $10 \%$ greater than baseline. Pace was monitored with a GPS watch. Cadence was cued by an auditory metronome and measured with both GPS watch and insoles. Repeated-measures ANOVA's examined the differences in average peak force, GPS-reported cadence, and insole-reported cadence between mile 1 and mile 2, and across the two cadence conditions.

\section{Results}

Cadence differences of $7.3 \%$ were observed between baseline and cadence sessions $(\mathrm{p}<0.001)$. A concurrent decrease in average peak force of $5.6 \%$ was demonstrated during the cadence run $(p<0.05)$. Average cadences measured by GPS watch and insoles were found to be the same at both baseline $(p=0.096)$ and during cadence $(p=0.352)$ sessions.

\section{Conclusion}

Increasing cadence by an average of $7 \%$ in an outdoor setting resulted in a decrease in peak force at two different time points during a 2.4-mile run. Furthermore, using a metronome for in-field cadence manipulation led to a change in cadence. This suggests that a metronome may be an effective tool to manipulate cadence for the purpose of decreasing peak impact force in an outdoor setting.

\footnotetext{
a Corresponding author:

Thomas W. Kernozek, PhD, FACSM,

University of Wisconsin, La

Crosse, Department of Health Professions, Physical Therapy Program,

La Crosse, WI 54601

kernozek.thom@uwlax.edu
} 


\section{Level of evidence}

$3 b$

\section{INTRODUCTION}

Within the U.S., the number of people running for exercise has dramatically increased over the past couple of decades. In 1990, five million people annually were reported to complete a road race. Nearly 30 years later, that number has reported to be 17.9 million. ${ }^{1}$ While this increase in recreational running has obvious health and wellness benefits for the general population, the relative risk of sustaining a running-related injury (RRI) should be considered. Lower extremity RRIs occur in an estimated $56 \%$ of recreational runners, with some suggesting this to be as high as $80 \%$. $^{2,3}$

Since RRIs are so prevalent, it is important to consider which factors may influence risk. While there have been a variety of proposed mechanisms for RRIs, high impact loading is often considered to be a factor. Davis, Bowser and Mullineaux ${ }^{4}$ examined 240 runners over a two-year period and reported that impact loads were greater in those runners who experienced an RRI compared to non-injured runners. They subsequently recommended that interventions aimed at decreasing the impact loads may be an effective strategy for reducing injury. ${ }^{4}$ In a systematic review by van der Worp, Vrielink and Bredeweg, ${ }^{5} 18$ studies were reviewed and showed that runners with higher loading rates were more likely to have injuries than those with lower rates.

Impact loads may play a large role in many RRIs; therefore, many clinicians and researchers have shifted their focus to remedies for reducing impact and loading rate as a form of injury prevention or to assist in return to running following injury. One method to alter such impact forces is to increase cadence. Increasing cadence while maintaining a consistent pace has been reported to be an effective way to immediately lower impact, thus reducing injury risk. ${ }^{6-8}$

Many studies to date have primarily been conducted in a controlled, laboratory setting utilizing motorized treadmills. While treadmills have been shown to produce biomechanically similar running patterns, they have the potential for changing spatiotemporal patterns. ${ }^{9}$ Tao, et al. ${ }^{10}$ reported an increased cadence, and decreased stance and swing time duration, when running on a level or inclined treadmill compared to running outdoors. In addition, outdoor running may produce notable variations in pace, cadence, and/or stride length during distance running events, as opposed to the constant pace imposed by the use of a treadmill. ${ }^{11}$ Therefore, to examine the efficacy of cadence manipulation used for recreational running in an etiologically valid scenario, how cadence influences impact forces should be evaluated within a natural outdoor running environment.

To produce a change in spatiotemporal gait parameters, a runner will require a feedback strategy to prompt an increase in cadence. The methods for administering such feedback include the use of concurrent (provided during a task) and terminal (provided at conclusion of task) feedback. ${ }^{12}$ According to Broker, et al., ${ }^{13}$ concurrent feedback is most effective for cyclical activities such as running and cycling. Such feedback, when provided in an auditory man- ner, produces the most desirable change in performance when provided immediately after the same event in each cycle of movement. ${ }^{13,14}$ In other words, to promote immediate change in running performance, an auditory stimulus should be given for each step taken. Additionally, alternative forms of concurrent feedback such as a visual stimulus are not feasible to provide to a runner in an open, outdoor environment. Previous studies utilizing an audio metronome in a laboratory environment have demonstrated changes to cadence in runners with the use of an external auditory cue.6,7 This simple feedback cue would be compatible for delivery by a wearable portable device such as a watch or smartphone used outdoors.

The purpose of this study was to examine the effects of increased cadence on peak impact force in an outdoor setting. It was hypothesized that an increased cadence would decrease peak ground reaction force. A secondary aim of this study was to explore the feasibility of using an auditory metronome as a stimulus cue to increase cadence during an outdoor run.

\section{METHODS}

\section{STUDY DESIGN}

This study utilized a repeated measures design, conducted on 15 recreational runners. Data were collected over a period of five weeks. The study protocol was approved by the University of Wisconsin-La Crosse Institutional Review Board and all participants provided informed consent prior to participation.

\section{PARTICIPANTS}

Fifteen male and female adult recreational runners were recruited for the study using convenience sampling of university students. Participants were recreational runners that ran an average of $14.8 \pm 8.4$ miles/week. The study excluded participants who reported a lower extremity injury in the prior three months, a history of lower extremity surgery, current lower extremity pain during running, or presence of a medical pathology that would cause difficulty running for up to one hour.

\section{INSTRUMENTS}

Loadsol insole sensors (novel gmbh, Munich, Germany) were placed inside each participant's typical running shoes bilaterally to measure peak force in Newtons (N). ${ }^{15}$ Peak force data were collected at $100 \mathrm{~Hz}$ and transmitted through Bluetooth connection to a $6^{\text {th }}$ generation iPod Touch (Apple, California, USA) using the pedoped Loadsol application (Version 1.4.72, novel gmbh, Munich, Germany). The Loadsol insoles have been shown to yield comparable peak force data compared to an instrumented treadmill for running (ICCs: 0.78-0.92) and reliable between sessions (ICCs: 0.88-0.93). ${ }^{16}$ Burns, et al. ${ }^{17}$ showed equally compelling data in support of Loadsol use to measure ground reaction forces 
by comparisons to hopping, walking and running on a force platform and an instrumented treadmill.

A Garmin Forerunner 25 GPS watch with Garmin Connect app (Version 4.5.1, Garmin International Inc., Olathe, KS) was used to determine running pace and cadence. Similar types of Garmin watches with GPS technology were validated as a measure of distance (absolute percent error: $0.74 \%$ ) and as a reliable measure of cadence (ICC: 0.93$).{ }^{18,19}$ The MetroTimer app (Version 3.3.2, ONYX Apps, United States) was used on the iPod Touch to provide auditory cadence cueing. Body mass measurements were taken using a digital scale.

\section{PROCEDURE}

Participants completed a questionnaire to collect training/ injury history and were then weighed. Participants were given up to 10 minutes to perform their self-selected warm up routine consisting of stretching and a short run no more than 0.25 mile. Following this warm up, Loadsol sensor insoles, GPS watch, and running belt containing iPod Touch were fitted for the participant. Loadsol sensor insoles were placed bilaterally in each participant's typical running shoes and then calibrated following the manufacturer's guidelines using the Loadsol application on the iPod touch. Calibration was completed following the procedure previously outlined by Peebles, et al. ${ }^{20}$ A quarter mile, warm up jog was then conducted to provide acclimation to this equipment.

Participants completed two separate 2.4-mile distance runs ("baseline" and "cadence" sessions) approximately one week apart. The selected road course was a straight stretch void of turns, with minimal elevation change $(<20$ feet), and minimal traffic. During a single run, participants ran 1.2 miles out, immediately turned around, and completed the same distance back to the starting point. This minimized the amount of turns on the straight, flat road available. The 2.4 mile distance was chosen for the run to allow for a comparison of miles 1 and 2 over time and to accommodate the acceleration up to a steady pace and acclimation to the auditory metronome cues.

For the baseline session, participants were instructed to run at a consistent, self-selected comfortable pace that they could maintain for up to an hour. The metronome was turned off for this run and no mention of running cadence was given to ensure that the run was completed at their typical cadence. Participants were given a GPS watch to monitor pace during this run. After the baseline session completion, cadence and pace data were gathered from the GPS watch to be utilized for setting up parameters for the cadence session scenario on a different day. Cadence and peak force data were collected from insoles. Based on previous treadmill cadence research, a $10 \%$ increase in cadence over individual baselines was used as the target for the cadence session scenario. 6,7

The cadence session was conducted within ten days of the baseline session to decrease the potential for training or fatigue effects. Participants were told to continue their normal training regimen between these sessions. Shoes, warm up time, and calibration procedures were consistent with their baseline session. The $10 \%$ increase in cadence was im- plemented using the MetroTimer metronome app on iPod Touch for the duration of the cadence session without an earbud or headphone on a quiet running course. Participants were reminded of their baseline pace and instructed to maintain that pace with use of GPS watch while also maintaining target cadence based on the metronome audio cues. A quarter mile jog was again provided to allow acclimation to the running pace and metronome cadence before beginning the session. Cadence was measured via both GPS watch and Loadsol insoles and examined at a later date.

\section{DATA MANAGEMENT AND ANALYSIS}

Peak force data were extracted from 20 right foot steps surrounding each quarter-mile increment and averaged, producing a total of eight force time series curves of the right stance phase during each run. Cadence was determined from insole data by obtaining right insole the peak-to-peak timing of the vGRF over the 20 steps for each quarter-mile increment and was reported as steps/minute (SPM). Since cadence is based typically on right and left foot contacts and only the right foot data were extracted for analysis, these times were divided by 2 . Similarly, peak forces were expressed in body weight (BW) for only these right steps. Peak force and insole cadence data were collapsed to an average over mile 1 and mile 2 separately for both baseline and cadence sessions. These data were analyzed in IBM SPSS Statistics version 25 (Armonk, NY, USA). To examine differences in average peak force, a repeated measures $2 \times 2$ ANOVA was performed on session (baseline, cadence sessions) and distance (mile 1, mile 2) (alpha was set to 0.05). An additional 2x2 ANOVA was performed on session (baseline, cadence sessions) and distance (mile 1, mile 2) variables to examine differences in insole-reported cadence. A third repeated measures ANOVA was performed on session (baseline, cadence sessions) and device (Insoles, GPS watch) to identify any differences between the two devices. Post hoc testing was performed using Bonferroni correction.

\section{RESULTS}

Fifteen participants ( 8 females, 7 males) with a mean age of 23.5 years (range 22-26) completed both baseline and cadence sessions. The reported average weekly mileage was 16.5 miles. A repeated measures ANOVA revealed a 5.6\% decrease in average peak force after cadence manipulation when compared to baseline $(\mathrm{p}<0.05)$ ( Figure 1). Peak force decreases were found to have medium effect size (Cohen's $\mathrm{d}=0.56$ ) (Table 1). During both the baseline and cadence session runs there was no difference in peak force during the first mile compared to the second mile $(p=0.202)$ and no interaction effect found between session and distance $(\mathrm{p}=0.13)$.

Mean and 95\% Confidence Intervals for average cadence expressed in steps per minute (SPM) on one lower extremity throughout Mile 1 and Mile 2 for both Baseline and Cadence Tests during outdoor running.

Results from the two-way ANOVA demonstrated insolemeasured cadence increased $7.3 \%$ from baseline to cadence sessions $(\mathrm{p}<0.001)$ (Figure 2). The effect size for the cadence increase was high (Cohen's $d=1.24$ ) (Table 2). Average ca- 
Table 1. Descriptive Statistics and ANOVA results for Insole-measured Cadence

\begin{tabular}{clcccc}
\hline & & Mean (SPM) & SD (SPM) & p-value & Effect Size (Cohen's d) \\
\hline \multirow{2}{*}{ Time } & Baseline test & 82.94 & 4.4 & \multirow{2}{*}{0.000} & 1.24 \\
& Cadence test & 89.03 & 4.33 & & \multirow{2}{*}{0.13} \\
\multirow{2}{*}{ Distance } & Mile 1 & 86.27 & 4.26 & 0.032 & \\
& Mile 2 & 85.71 & 4.34 & & \\
\hline
\end{tabular}

$\mathrm{SPM}=$ unilateral steps per minute

dence was found to decrease from mile 1 to mile 2 by 0.56 steps per minute $(\mathrm{p}=0.032)$. However, there was no interaction effect found between session and distance $(\mathrm{p}=0.597)$.

Mean and 95\% Confidence Intervals for peak vertical ground reaction force (vGRF) expressed in multiples of bodyweight (BW) between Mile 1 and Mile 2 for Baseline and Cadence Tests during outdoor running.

When comparing the average cadence measured by the insoles against the cadence measured by the GPS watch, the two-way ANOVA results revealed no difference in measurement between the devices ( $\mathrm{p}=0.096$ ), yet an overall increase in average cadence remained between sessions $(p<0.001)$, confirming that both devices were able to detect a similar percent change in cadence from baseline to cadence sessions (Figure 3) (Table 3). No interaction effect was determined between session and distance $(\mathrm{p}=0.928)$.

Mean and 95\% Confidence Intervals for average cadence expressed in steps per minute (SPM) on one lower extremity throughout Baseline and Cadence Tests. Data compared cadence measured between Garmin GPS watch (Watch) and Loadsol insole sensors (Insole) during outdoor running.

\section{DISCUSSION}

The purpose of this study was twofold: 1) to determine the effect of cadence modification on peak impact force during an outdoor run, and 2) to examine the immediate response and magnitude of cadence modification through the use of a metronome as an auditory stimulus during a second session. It was hypothesized that as cadence increased, peak impact forces would decrease. It was additionally hypothesized that use of a metronome would produce a higher cadence during the run.

In support of these hypotheses, participants demonstrated a substantial increase in cadence by $7.3 \%$ using a metronome for outdoor running with a concurrent decrease in average peak impact force by $5.6 \%$. One possible explanation for this change in lower extremity impact could be due to a change in foot strike pattern that may occur from the manipulation of the spatiotemporal factors associated with gait, however this was not directly measured or quantified. Increasing cadence has been shown to promote a change from a rearfoot strike pattern to a mid- or forefoot strike, which may result in decreased vertical loading between $0.3-1.3$ body weights (BW). ${ }^{21,22}$ A change from rearfoot to forefoot strike may have other beneficial effects including decreasing knee joint contact forces by an average of $1.2 \mathrm{BW}^{23}$ and decreasing patellofemoral joint stress by an

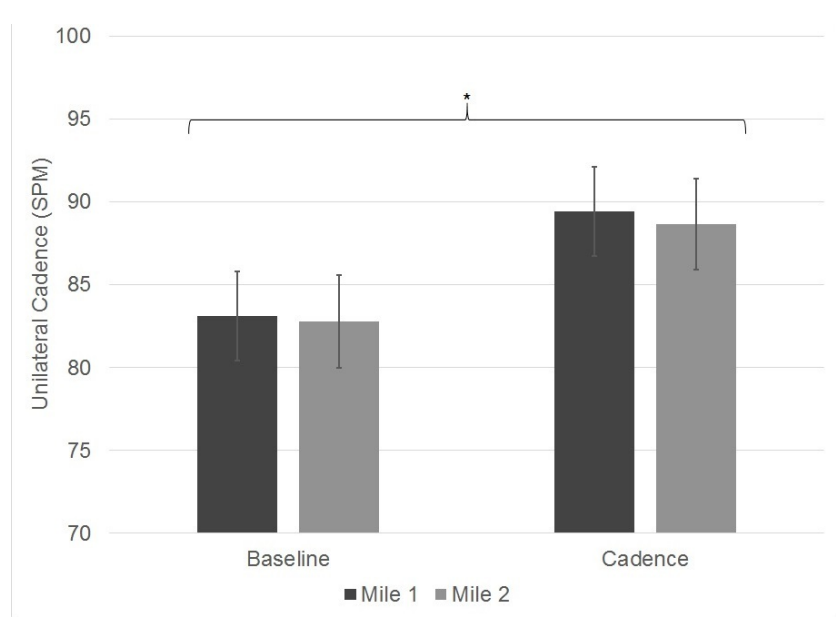

Figure 1. Mean and 95\% Confidence Intervals for average cadence expressed in steps per minute (SPM) on one lower extremity throughout Mile 1 and Mile 2 for both Baseline and Cadence Tests during outdoor running

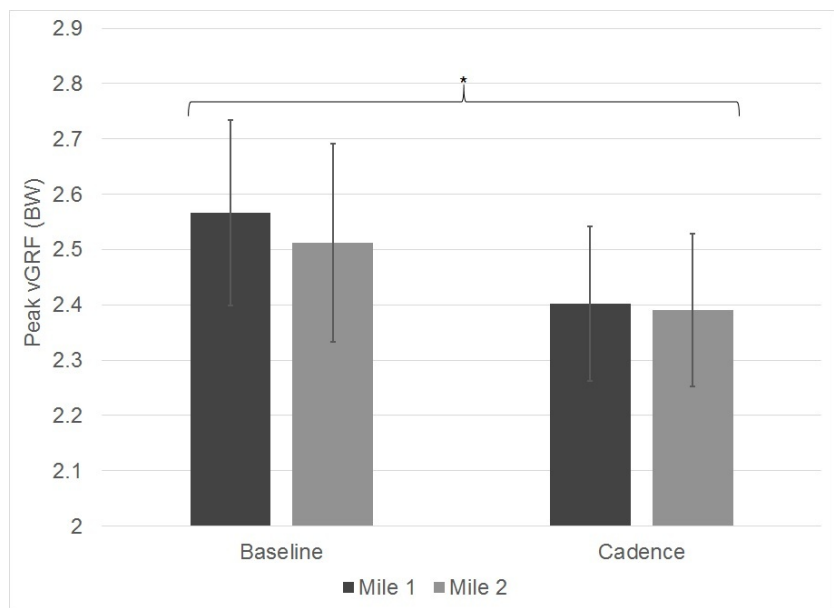

Figure 2. Mean and 95\% Confidence Intervals for peak vertical ground reaction force (vGRF) expressed in multiples of bodyweight (BW) between Mile 1 and Mile 2 for Baseline and Cadence Tests during outdoor running

average of $27 \% .{ }^{24}$ However, a forefoot strike may increase stress and loading at the Achilles tendon, ankle, and plantar 
Table 2. Descriptive Statistics and ANOVA results for Peak GRF

\begin{tabular}{|c|c|c|c|c|c|}
\hline & & Mean (BW) & $\mathrm{SD}(\mathrm{BW})$ & $\mathrm{p}$-value & Effect Size (Cohen's d) \\
\hline \multirow{2}{*}{ Time } & Baseline test & 2.539 & 0.267 & \multirow{2}{*}{0.029} & \multirow{2}{*}{0.56} \\
\hline & Cadence test & 2.396 & 0.214 & & \\
\hline \multirow[b]{2}{*}{ Distance } & Mile 1 & 2.484 & 0.222 & \multirow[b]{2}{*}{0.202} & \multirow[b]{2}{*}{0.146} \\
\hline & Mile 2 & 2.451 & 0.229 & & \\
\hline
\end{tabular}

$\mathrm{GRF}=$ ground reaction force, $\mathrm{BW}=$ peak force normalized by multiples of individual body weight

Table 3. Descriptive Statistics and ANOVA results for Cadence between GPS and Insole

\begin{tabular}{clcccc}
\hline & & Mean (SPM) & SD (SPM) & p-value & Effect Size (Cohen's d) \\
\hline \multirow{2}{*}{ Time } & Baseline test & 82.75 & 4.35 & \multirow{2}{*}{0.000} & 1.43 \\
& Cadence test & 88.81 & 4.11 & & 0.08 \\
\hline \multirow{2}{*}{ Device } & Insole & 85.99 & 4.28 & 0.067 & 0.12 \\
& Watch & 85.57 & 4.12 & & \\
\end{tabular}

Watch $=$ Garmin GPS watch, Insole= Loadsol insole sensor, SPM= unilateral steps per minute

surface of the foot. ${ }^{25-27}$ Therefore, the benefits of changing foot strike pattern may need to be considered alongside the potential negative effects when implementing a gait modification technique.

Because of the maintained running speed between conditions, these findings may also be partially explained by the relationship between cadence and stride length in running. Since running speed is a product of cadence and stride length, an appreciable change in cadence at a constant running speed should result in a proportional reduction in stride length as a typical change. ${ }^{22}$ Specifically, decreasing a runner's stride length by $10 \%$ has been reported to have beneficial effects on knee kinetics, including decreased contact forces at the patellofemoral joint by an average of $14.9 \%$, decreased loading rate by $13 \%$, and decreased impulse per kilometer by $4.5 \% .^{28,29}$

Participants in this study were asked to increase cadence by $10 \%$ and, on average, were able to achieve approximately $7 \%$ above their preferred. Yet, this change was sufficient to produce a decrease in peak impact force between cadence conditions. The results of this study support data reported by Heiderscheit, et al., ${ }^{6}$ where both $5 \%$ and $10 \%$ increases in cadence decreased energy absorption required at the knee by approximately $20 \%$ and $40 \%$, respectively, as well as decreased energy absorbed at the hip (by $~ 57 \%$ ) with $10 \%$ increase in cadence. Other studies suggest comparable benefits at the patellofemoral joint, with an average of $14 \%$ lower contact forces, ${ }^{7}$ and at the foot, with a decrease in overall plantar loading between $2.4-8.0 \%{ }^{26}$ Similarly, Willy, et al. ${ }^{30}$ described changes in multiple lower extremity loading variables at just $7.5 \%$ increase in cadence. These changes included a decrease in average vertical loading rate by $17.9 \%$ as well as instantaneous vertical loading rate by $18.9 \% .30$ In contrast, Hobara, et al. ${ }^{31}$ reported a minimum of $15 \%$ increase in cadence to produce changes in lower extremity loading variables such as vertical loading rate and vertical

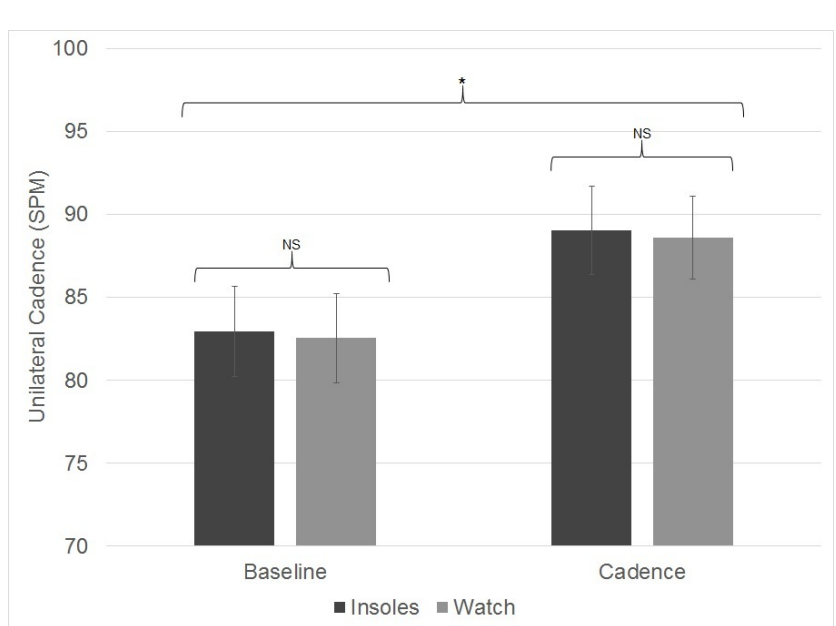

Figure 3. Mean and 95\% Confidence Intervals for average cadence expressed in steps per minute (SPM) on one lower extremity throughout Baseline and Cadence Tests. Data compared cadence measured between Garmin GPS watch (Watch) and Loadsol insole sensors (Insole) during outdoor running

impact peak. However, some have suggested increases above $10 \%$ may not be necessary to produce the desired lower extremity benefits and may negatively increase perceived exertion and metabolic cost during running. 6,30

The results demonstrated in the current study may have implications for using cadence modifications to reduce peak impact force as well as promote the suggested benefits on other lower extremity loading variables during an outdoor run. Previous studies had suggested high impact forces are associated with development of common RRIs, such as me- 
dial tibial stress syndrome, ${ }^{32,33}$ Achilles tendinopathy, 33,34 plantar fasciitis, 33,35 and patellofemoral pain syndrome. ${ }^{33,36}$ However, a prospective study by Szymanek, et al. ${ }^{37}$ discovered no association between a runner's preferred cadence and development of lower extremity overuse injuries. Additionally, a recent meta-analysis by Vannatta, et al. ${ }^{38}$ reported a conflicting association between peak impact force and RRIs. In the same study, they reported limited evidence relating decreased step rate to increased risk of shinrelated injuries. ${ }^{38}$ Further investigation into the use of cadence modification for the treatment or prevention of pain and RRIs appears to be warranted as was suggested in their review.

Altering patient cadence with the goal of reducing impact forces may be feasibly accomplished in a clinical setting using a treadmill. ${ }^{6-8}$ The results of the present study demonstrate that similar cadence modifications can be feasibly implemented within an outdoor setting with the use of wearable technology and metronome feedback. Cadence measured through the GPS watch device was similar to the insole-measured cadence, indicating this commercially available technology may provide an effective method for measuring running cadence during indoor or outdoor gait retraining. This may open opportunities to provide running with cadence training protocols to be used in an outdoor running environment. This may also be a beneficial adjunct to training for military personnel where it has been reported that $25 \%$ of male and $50 \%$ of female military recruits suffer an injury related to their training, with $60-80 \%$ of those injuries being considered an overuse lower extremity injury. ${ }^{39}$ Since much of military training is completed in an outdoor setting, use of a metronome and GPS watch may provide a feasible alternative for in-field feedback and cueing of cadence.

\section{LIMITATIONS}

This study had several limitations that may affect the interpretation of the findings presented here. First, the study was performed on only healthy runners who did not have a history of lower extremity injuries. Therefore, these results should not be considered to be representative of an injured running population. Further studies should examine if injured runners behave in a similar manner for cadence training in an outdoor setting. Second, although every attempt was made to control many of the elements in our outdoor setting, certain environmental variables were sub- ject to change between baseline and cadence session procedures such as wind speed, temperature, and amount of vehicle traffic. These variables, even when minimized, may have an effect on the within-subject changes that were reported here. Third, the study consisted of an intervention performed with a small sample size of only 15 participants which may limit the power of statistical inferences. Further studies should include a greater number of participants to replicate these findings. Finally, while not a direct aim of this study, there was no long-term assessment of cadence retention without GPS watch and metronome use. Therefore, it is unknown whether these acute changes in cadence shown here through use of a metronome would be retained. However, Willy, et al. ${ }^{30}$ suggested maintenance of increased step frequency may be effective for a 30-day duration. Despite this, future research should examine for retention of cadence modifications over an extended period, as well as the potential for use of a fading feedback schedule, to promote long-term changes without reliance on concurrent feedback.

\section{CONCLUSION}

Using a metronome to increase cadence in an outdoor setting may be an effective way to reduce impact forces during running in an outdoor setting. Furthermore, using wearable technology that can provide cadence feedback via auditory cues may produce changes to cadence outside of the lab. Participants were immediately able to make a $7 \%$ average increase in cadence within one session using a metronome, suggesting that this may be an effective tool for manipulating cadence during outdoor running. While further research is indicated to examine the cadence effects on RRI incidence and for "in the field" training, these findings demonstrate a possibility for cadence alterations to be feasible in outdoor settings.

\section{CONFLICTS OF INTEREST}

All authors report no conflict of interest associated with this project.

Submitted: November 09, 2020 CDT, Accepted: February 21, 2021 CDT 


\section{REFERENCES}

1. Running USA Releases Latest U.S. Running Trends Report. https://www.runningusa.org/RUSA/Research/ Recent_Reports/Road_Race_Trends/RUSA/News/2018/ U.S. Road Race Participation Numbers Hold Stead $\mathrm{y}$ for_2017.aspx?hkey=5dfc2cef-24c1-47ae-acfb-c7069 9455df6. Accessed April 20, 2020.

2. van Gent RN, Siem D, van Middelkoop M, van Os AG, Bierma-Zeinstra SMA, Koes BW. Incidence and determinants of lower extremity running injuries in long distance runners: a systematic review. Br J Sports Med. 2007;41(8):469-480; discussion 480. doi:10.113 6/bjsm.2006.033548

3. Jacobs SJ, Berson BL. Injuries to runners: a study of entrants to a 10,000 meter race. Am J Sports Med. 1986;14(2):151-155. doi:10.1177/03635465860140021 1

4. Davis IS, Bowser BJ, Mullineaux DR. Greater vertical impact loading in female runners with medically diagnosed injuries: a prospective investigation. Br J Sports Med. 2016;50(14):887-892. $\underline{\mathrm{d}}$ oi:10.1136/bjsports-2015-094579

5. van der Worp H, Vrielink JW, Bredeweg SW. Do runners who suffer injuries have higher vertical ground reaction forces than those who remain injuryfree? A systematic review and meta-analysis. $\mathrm{Br} \mathrm{J}$ Sports Med. 2016;50(8):450-457. doi:10.1136/bjsport s-2015-094924

6. Heiderscheit BC, Chumanov ES, Michalski MP, Wille CM, Ryan MB. Effects of step rate manipulation on joint mechanics during running. Med Sci Sports Exerc. 2011;43(2):296-302. doi:10.1249/MSS.0b013e3 181ebedf4

7. Lenhart RL, Thelen DG, Wille CM, Chumanov ES, Heiderscheit BC. Increasing running step rate reduces patellofemoral joint forces. Med Sci Sports Exerc. 2014;46(3):557-564. doi:10.1249/MSS.0b013e3182a78 c3a

8. Schubert AG, Kempf J, Heiderscheit BC. Influence of stride frequency and length on running mechanics: A systematic review. Sports Health. 2013;6(3):210-217. doi:10.1177/1941738113508544

9. Van Hooren B, Fuller JT, Buckley JD, et al. Is motorized treadmill running biomechanically comparable to overground running? A systematic review and meta-analysis of cross-over studies. Sports Med. 2020;50(4):785-813. doi:10.1007/s40279-019-01 237-z
10. Tao H, Joyce L, Kozak B, Luiken J, Wendt N. Spatiotemporal comparison of overground and treadmill running with pressure sensor insoles in division I collegiate runners. Int J Sports Phys Ther. 2019;14(5):731-739.

11. Hanley B, Mohan AK. Changes in gait during constant pace treadmill running. J Strength Cond Res. 2014;28(5):1219-1225. doi:10.1519/JSC.0b013e3182a3 $\underline{8796}$

12. Salmoni AW, Schmidt RA, Walter CB. Knowledge of results and motor learning: a review and critical reappraisal. Psychol Bull. 1984;95(3):355-386.

13. Broker JP, Gregor RJ, Schmidt RA. Extrinsic feedback and the learning of kinetic patterns in cycling. J Appl Biomech. 1993;9(2):111-123. doi:10.112 3/jab.9.2.111

14. Baudry L, Leroy D, Thouvarecq R, Choller D. Auditory concurrent feedback benefits on the circle performed in gymnastics. J Sports Sci. 2006;24(2):149-156. doi:10.1080/02640410500130979

15. Hill M, Hoena B, Kilian W, Odenwald S. Wearable, modular and intelligent sensor laboratory. Procedia Eng. 2016;147:671-676. doi:10.1016/j.proeng.2016.0 $\underline{6.270}$

16. Renner KE, Williams DSB, Queen RM. The reliability and validity of the loadsol ${ }^{\circledR}$ under various walking and running conditions. Sensors. 2019;19(2). doi:10.3390/s19020265

17. Burns GT, Deneweth Zendler J, Zernicke RF. Validation of a wireless shoe insole for ground reaction force measurement. J Sports Sci. 2019;37(10):1129-1138. doi:10.1080/02640414.2018.1 $\underline{545515}$

18. Adamakis M. Comparing the validity of a GPS monitor and a smartphone application to measure physical activity. J Mob Technol Med. 2017;6(2):28-38.

19. Adams D, Pozzi F, Carroll A, Rombach A, Zeni J. Validity and reliability of a commercial fitness watch for measuring running dynamics. J Orthop Sports Phys Ther. 2016;46(6):471-476. doi:10.2519/jospt.2016.639 $\underline{1}$

20. Peebles AT, Maguire LA, Renner KE, Queen RM. Validity and repeatability of single-sensor loadsol insoles during landing. Sensors. 2018;18(12):4082. do i: $10.3390 / \mathrm{s} 18124082$ 
21. Almeida MO, Davis IS, Lopes AD. Biomechanical differences of foot-strike patterns during running: A systematic review with meta-analysis. J Orthop Sports Phys Ther. 2015;45(10):738-755. doi:10.2519/jospt.20 15.6019

22. Lieberman DE, Warrener AG, Wang J, Castillo ER. Effects of stride frequency and foot position at landing on braking force, hip torque, impact peak force and the metabolic cost of running in humans. $J$ Exp Biol. 2015;218(Pt 21):3406-3414. doi:10.1242/je b. 125500

23. Kulmala J-P, Avela J, Pasanen K, Parkkari J. Forefoot strikers exhibit lower running-induced knee loading than rearfoot strikers. Med Sci Sports Exerc. 2013;45(12):2306-2313. doi:10.1249/MSS.0b013e3182 9efcf7

24. Vannatta CN, Kernozek TW. Patellofemoral joint stress during running with alterations in foot strike pattern. Med Sci Sports Exerc. 2015;47(5):1001-1008. d oi:10.1249/MSS.0000000000000503

25. Lyght M, Nockerts M, Kernozek TW, Ragan R. Effects of foot strike and step frequency on achilles tendon stress during running. J Appl Biomech. 2016;32(4):365-372. doi:10.1123/jab.2015-0183

26. Wellenkotter J, Kernozek TW, Meardon S, Suchomel T. The effects of running cadence manipulation on plantar loading in healthy runners. Int J Sports Med. 2014;35(9):779-784. doi:10.1055/s-0 033-1363236

27. Rooney BD, Derrick TR. Joint contact loading in forefoot and rearfoot strike patterns during running. $J$ Biomech. 2013;46(13):2201-2206. doi:10.1016/j.jbiom ech.2013.06.022

28. Bowersock CD, Willy RW, DeVita P, Willson JD. Independent effects of step length and foot strike pattern on tibiofemoral joint forces during running. $J$ Sports Sci. 2017;35(20):2005-2013. doi:10.1080/02640 $\underline{414.2016 .1249904}$

29. Boyer ER, Derrick TR. Lower extremity joint loads in habitual rearfoot and mid/forefoot strike runners with normal and shortened stride lengths. J Sports Sci. 2018;36(5):499-505. doi:10.1080/02640414.2017.1321 $\underline{775}$
30. Willy RW, Buchenic L, Rogacki K, Ackerman J, Schmidt A, Willson JD. In-field gait retraining and mobile monitoring to address running biomechanics associated with tibial stress fracture. Scand J Med Sci Sports. 2016;26(2):197-205. doi:10.1111/sms.12413

31. Hobara H, Sato T, Sakaguchi M, Sato T, Nakazawa $\mathrm{K}$. Step frequency and lower extremity loading during running. Int J Sports Med. 2012;33(4):310-313. doi:1 $\underline{0.1055 / \mathrm{s}-0031-1291232}$

32. Popp KL, McDermott W, Hughes JM, Baxter SA, Stovitz SD, Petit MA. Bone strength estimates relative to vertical ground reaction force discriminates women runners with stress fracture history. Bone. 2017;94:22-28. doi:10.1016/i.bone.2016.10.006

33. Lopes AD, Hespanhol LC, Yeung SS, Costa LOP. What are the main running-related musculoskeletal injuries? Sports Med Auckl Nz. 2012;42(10):891-905. d oi:10.1007/BF03262301

34. Lorimer AV, Hume PA. Achilles tendon injury risk factors associated with running. Sports Med Auckl NZ. 2014;44(10):1459-1472. doi:10.1007/s40279-014-020 9-3

35. Pohl MB, Hamill J, Davis IS. Biomechanical and anatomic factors associated with a history of plantar fasciitis in female runners. Clin J Sport Med. 2009;19(5):372-376. doi:10.1097/JSM.0b013e3181b8c $\underline{270}$

36. Thijs Y, De Clercq D, Roosen P, Witvrouw E. Gaitrelated intrinsic risk factors for patellofemoral pain in novice recreational runners. Br J Sports Med. 2008;42(6):466-471. doi:10.1136/bjsm.2008.046649

37. Szymanek EB, Miller EM, Weart AN, Morris JB, Goss DL. Is step rate associated with running injury incidence? Int J Sports Phys Ther. 2020;15(2):221-228.

38. Vannatta CN, Heinert BL, Kernozek TW. Biomechanical risk factors for running-related injury differ by sample population: A systematic review and meta-analysis. Clin Biomech. 2020;75:104991. doi:1 $\underline{0.1016 / j . c l i n b i o m e c h .2020 .104991}$

39. Bullock SH, Jones BH, Gilchrist J, Marshall SW. Prevention of physical training-related injuries recommendations for the military and other active populations based on expedited systematic reviews. Am J Prev Med. 2010;38(1 Suppl):S156-181. doi:10.101 6/j.amepre.2009.10.023 